\title{
Application of Distance Geometry to Tracing Coupler Curves of Pin-Jointed Linkages*
}

\author{
Nicolás Rojas ${ }^{\dagger}$ and Federico Thomas \\ Institut de Robòtica i Informàtica Industrial (CSIC-UPC) \\ Llorens Artigas 4-6, 08028 Barcelona, Spain \\ \{nrojas, fthomas\}@iri.upc.edu
}

\begin{abstract}
In general, high-order coupler curves of single-degree-offreedom plane linkages cannot be properly traced by standard predictor-corrector algorithms due to drifting problems and the presence of singularities. Instead of focusing on finding better algorithms for tracing curves, a simple method that first traces the configuration space of planar linkages in a distance space and then maps it onto the mechanism workspace, to obtained the desired coupler curves, is proposed. Tracing the configuration space of a linkage in the proposed distance space is simple because the equation that implicitly defines this space can be straightforwardly obtained from a sequence of bilaterations, and the configuration space embedded in this distance space naturally decomposes into components corresponding to different combinations of signs for the oriented areas of the triangles involved in the bilaterations. The advantages of this two-step method are exemplified by tracing the coupler curves of a double butterfly linkage.
\end{abstract}

Keywords: Linkages, configuration spaces, coupler curves, Distance Geometry, double butterfly linkage.

\section{Introduction}

The mechanisms considered under the title linkwork, linkages, or articulated systems are plane mechanisms involving turning pairs only. That is, sets of plane links articulated through pins. For mechanisms of this type, the equation of the curve generated by an arbitrary point on the mechanism - the tracer point — can be obtained by solving a finite number of simultaneous equations expressing constancy of distance between pin centers which include the tracer point. Then, the coordinates of all moving pin points, other than those of the tracer, can be seen as unknowns. If we only considered single-degree-of-freedom mechanisms, the number of independent quadratic equations will be one fewer than the number of unknowns. The curve generated by the tracer point - usually known as a coupler curve - is, therefore, the eliminant of these equations. This reasoning permits to conclude that the curve generated by any point on a plane

${ }^{*}$ Some of the ideas contained in this paper were already presented at the ASME 2011 International Design Engineering Technical Conferences \& Computers and Information in Engineering Conference IDETC/CIE 2011, August 28-31, 2011, Washington, DC, USA

${ }^{\dagger}$ Corresponding author. pin-jointed mechanism possessing a finite number of links of finite size is necessarily algebraic [1]. The same result can be attained, in a more compact way, by computing the eliminant of the set of independent loop equations [2], [3], [4]. All coupler curves can be seen as a group of manifold curves joined through singular points usually classified in kinematics as crunodes and cusps [5, 6].

The problem of tracing a coupler curve is essentially that of connecting sampled points to give rise to its graph. Sampling a coupler curve is not a difficult task compared to that of connecting the samples, mainly for high-order coupler curves. Continuation methods are one of the major approaches reported in the literature to solve this problem [7]. Since, in our case, the curves to be traced are algebraic, polynomial continuation can be used [8]. These methods are global, that is, they are able to trace all the connected components of a coupler curve but, depending on the application, one does not need to trace all components, but rather one of them starting from a given point. Actually, this is the encountered problem when simulating the motion of a plane mechanism [9]. In this case, a very popular method is the so-called predictor-corrector method [10]. It consists of two major stages. In the first stage, called the predictor step, a point in the tangent line to the curve at the current given point is estimated [Fig. 1(a)]. In the second stage, the corrector step, the predicted point is adjusted onto the curve, using typically a Newton-like method, to get a new point of the curve [Fig. 11 b)]. In the case of closed curves, a third stage, called the filling step, is implemented to avoid overlaps. The predictor-corrector algorithm is simple to implement and hence its popularity. Unfortunately, it exhibits, in general, the undesirable phenomenon known as drifting in which the procedure fails to keep moving along a given branch of the curve and drift to another [Fig. 1(c)]. In most dramatic cases this might even lead to cycling [Fig. 11d)]. This drawback can be resolved using more sophisticated mathematical tools, than the first order approximations used in standard predictor-corrector algorithms, such as Runge-Kutta or Adam's method [11]. Another important drawback of predictor-corrector algorithms arises when the plane curve to be traced has singular points because the tangent is undefined at them. An approach to overcome this issue is by first computing the singular points with symbolic processing techniques for then use them as starting points of a predictor-corrector algorithm. Unfortu- 


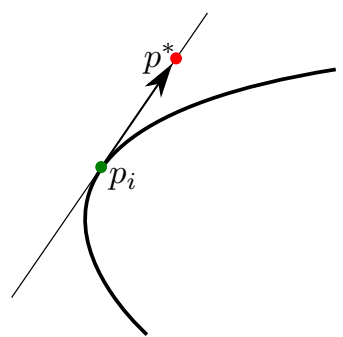

(a)

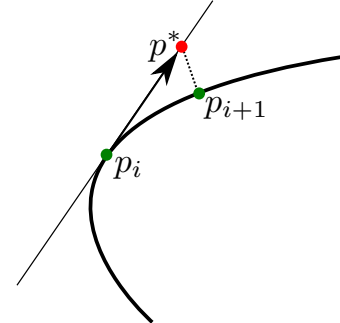

(b)

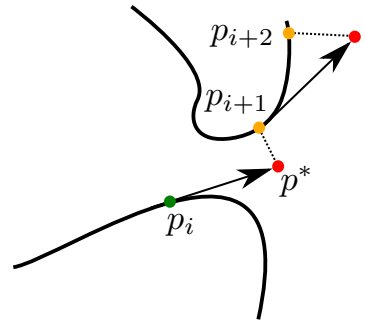

(c)

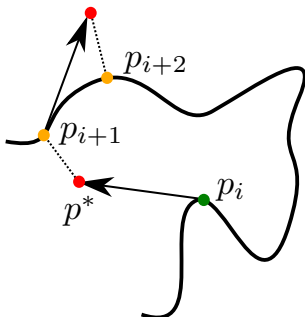

(d)

Figure 1: (a) In the predictor step, a point $p^{*}$ in the tangent line to the curve at the current point $p_{i}$ is estimated. (b) In the corrector step, the predicted point $p^{*}$ is adjusted onto the curve producing a new point $p_{i+1}$. (c) The drifting problem. (d) The cycling problem

nately, all these methods, generally based on independent loop equations, have only been presented for particular families of mechanisms [12, 13, 14, 15]. A simpler and more elegant alternative, valid only for plane curves, is the use of derivative-free methods such as the Morgado-Gomes false position numerical method [16].

The possibility of drifting, cycling, and having problems with singular points, increases dramatically with the number of independent kinematic loops of the linkage because the order of the coupler curves to be traced grows exponentially with it. For example, while the coupler curves of the well-known single-loop four-bar linkage are of the sixth order [17], that of the three-loop double butterfly linkage can be up to the forty-eighth order [18].

In this paper, instead of focusing on a better algorithm for tracing coupler curves able to deal with all mentioned problems in the workspace of the mechanism, a Distance Geometry approach that first traces the configuration space of the mechanism in a distance space and then maps it onto the workspace to obtained the desired coupler curves is proposed. To get an intuitive idea of this approach and its advantages, Section 2 presents the main clues without going into mathematical details. Then, Section 3 presents the basic mathematical tools to formalize them, and Section 4 concentrates on the case study of tracing the coupler curves of the double butterfly linkage. An example is then presented in Section 5. Section 6 discusses how the presented approach can be applied to other single-degree-of-freedom linkages and, finally, Section 7 summarizes the main contributions.

\section{Overview of the proposed approach}

A linkage configuration is given by a set of parameters uniquely specifying the position of each of its links. The configuration space of a linkage is thus simply the set of all its configurations. Then, since all points of a single-degree-offreedom linkage trace plane curves which can readily be expressed in terms of the configuration parameters of the linkage itself, an alternative approach, other than directly tracing coupler curves in the linkage workspace, naturally arises: first trace the configuration space of the mechanism and then compute the desired curves from it. To exemplify this idea, let us consider the four-bar linkage in Fig. 2(a). The cou- pler curve traced by any point linked to one of its bars, while taking the opposite bar as fixed, are algebraic curves of the sixth order; i.e., a straight line will cut it in not more than six points [17] [Fig. 2(b)]. The configuration space of this linkage can be easily derived by expressing the location of the line segments $\overline{P_{1} P_{3}}$ and $\overline{P_{2} P_{4}}$ as a function of $\theta_{1}$ and $\theta_{2}$, respectively, and imposing the distance between $P_{3}$ and $P_{4}$ as closure condition [19, pp. 26-27]. Thus, all possible configurations of this linkage defines a curve in the space defined by $\theta_{1}$ and $\theta_{2}$. Unfortunately, this idea cannot be applied, in general, to multi-loop linkages. Actually, the valid configurations of a multi-loop linkage is usually represented by the solution set of an independent set of its vector loop equations [2], [3], [4]. This requires introducing a variable for each link representing its orientation with respect to the fixed link. For the case of the four-bar linkage, its vector loop equation defines a one-dimension variety in the space defined by $\left\{\theta_{1}, \theta_{2}, \theta_{3}\right\}$ which seems quite complicated for such a simple linkage [Fig. 2(c)]. Alternatively, the configuration space of a four-bar linkage can be represented by a single distance variable, for example the distance between $P_{2}$ and $P_{3}$, provided that the sign of the oriented areas of the triangles $\triangle P_{1} P_{2} P_{3}$ and $\triangle P_{2} P_{4} P_{3}$ are given [Fig. 2](d)]. Besides an important reduction in the dimensionality of the problem, the configuration space of the linkage is thus decomposed into up to four components, one for each combination of signs for the two oriented areas. Most importantly, this idea of using distances and signs of oriented areas can be applied to characterize the configuration spaces of arbitrary multi-loop linkage. For example, let us consider the three-loop linkage, commonly known as double butterfly linkage, depicted in Fig. 3 a). Using the standard formulation based in vector loop equations, its configuration space is determined by the solution set of a system of six scalar equations in the space defined by $\left\{\theta_{1}, \ldots, \theta_{7}\right\}$. Using the Distance Geometry approach proposed in this paper, we will show how this configuration space can be characterized by a plane curve in the space defined by the lengths of $\overline{P_{1} P_{6}}$ and $\overline{P_{2} P_{4}}$, and how this curve is decomposed into 16 components, one for each combination of signs of the oriented areas of the triangles $\triangle P_{2} P_{4} P_{10}, \triangle P_{1} P_{3} P_{6}, \triangle P_{1} P_{6} P_{5}$ and $\triangle P_{4} P_{9} P_{7}$. This decomposition, together with the reduction of the dimensionality of the ambient space from 7 to 


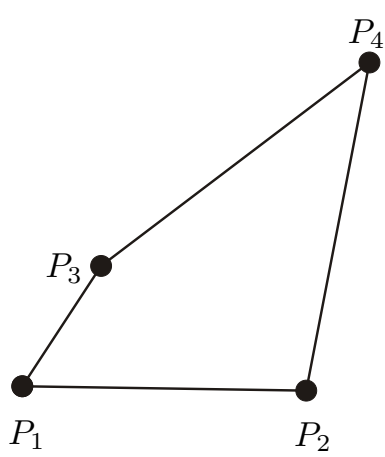

(a)

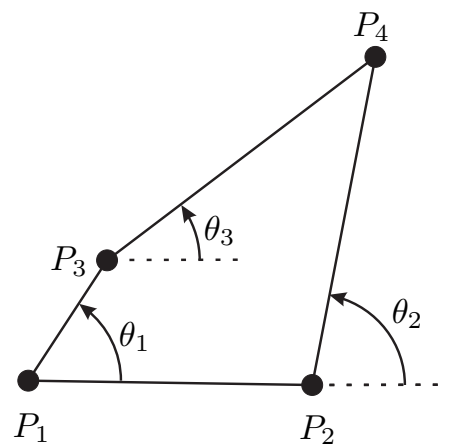

(c)

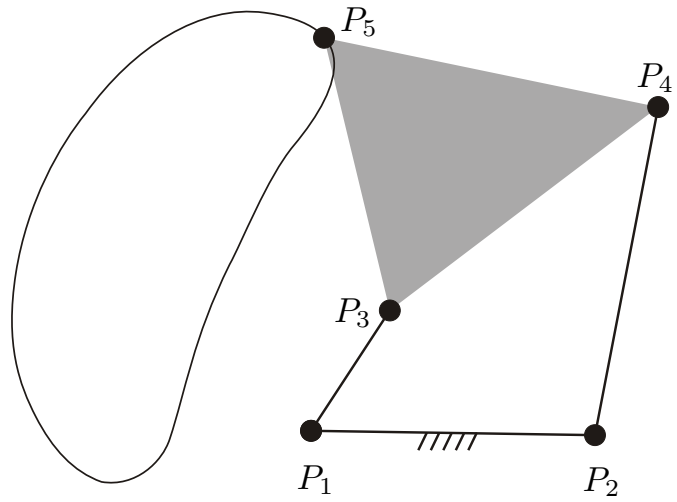

(b)

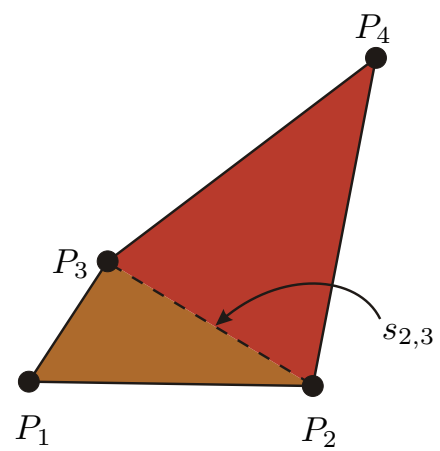

(d)

Figure 2: (a) A four-bar linkage and (b) the coupler curve traced by a point affixed to one of its bars while taking the opposite one as fixed. (c) Any coupler curve generated by this linkage can be expressed in terms of its configuration space which can be represented by a one-dimension variety in the space defined by $\left\{\theta_{1}, \theta_{2}, \theta_{3}\right\}$, or by $\left\{\theta_{1}, \theta_{2}\right\}$ if the distance constraint between $P_{3}$ and $P_{4}$ is used as closure condition instead of the standard loop equation. (d) Alternatively, this configuration space can be represented by value ranges of a single variable, $s_{2,3}$, one range for each combination of signs of the oriented areas of the triangles $\triangle P_{1} P_{2} P_{3}$ and $\triangle P_{2} P_{4} P_{3}$.

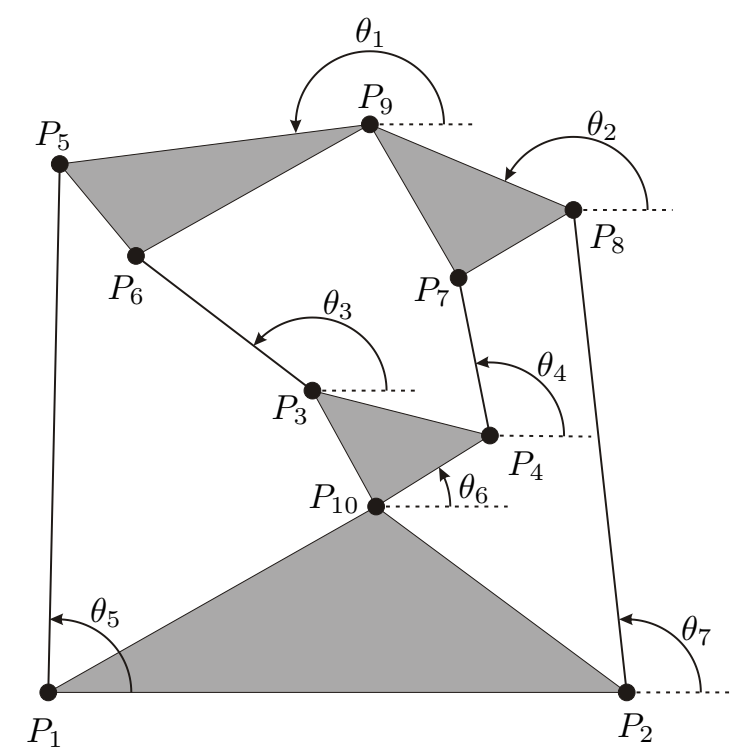

(a)

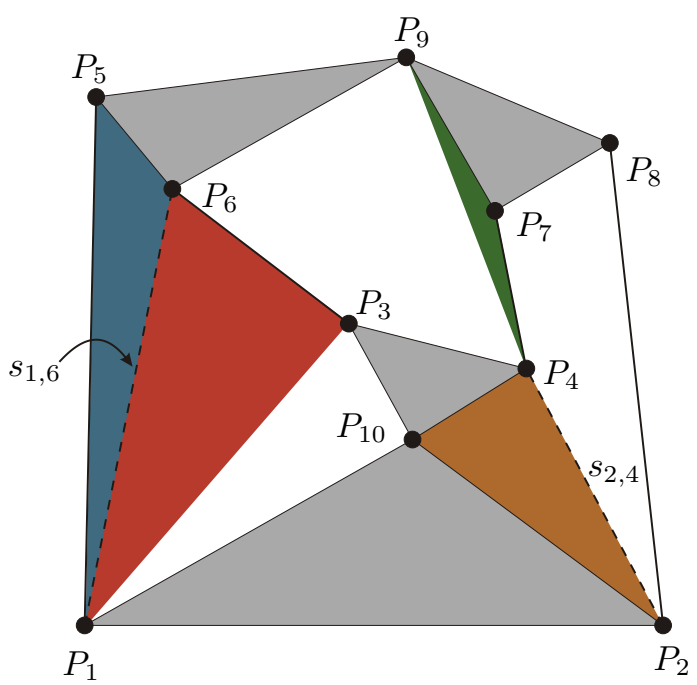

(b)

Figure 3: (a) Using the standard vector loop formulation, the configuration space of a double butterfly linkage can be represented by a one-dimensional variety in the space defined by $\left\{\theta_{1}, \ldots, \theta_{7}\right\}$. (b) Alternatively, using the proposed approach, this configuration space can be represented by a one-dimensional variety in the space defined by $\left\{s_{1,6}, s_{2,4}\right\}$ which can be decomposed into 16 components, one for each combination of signs of the oriented areas of the triangles $\triangle P_{2} P_{4} P_{10}$, $\triangle P_{1} P_{3} P_{6}, \triangle P_{1} P_{6} P_{5}$ and $\triangle P_{4} P_{9} P_{7}$. 
2 , greatly simplifies the process of tracing the configuration space of this linkage while retaining, at the same time, the geometric flavor of the problem contrarily to what happens to the fully algebraic current approaches.

It can be argued that the proposed approach belongs to the set of methods based on the idea of dividing the linkage's circuits - i.e., subsets of the configuration space in which a configuration can be continuously transformed to another one- into segments connected by special points because the linkage's circuits are also divided into components. However, in the proposed technique the special points those configuration space points where a given oriented area vanishes - have not to be calculated beforehand.

The next section presents the necessary mathematical tools to formalize the proposed approach.

\section{Distances in strips of triangles}
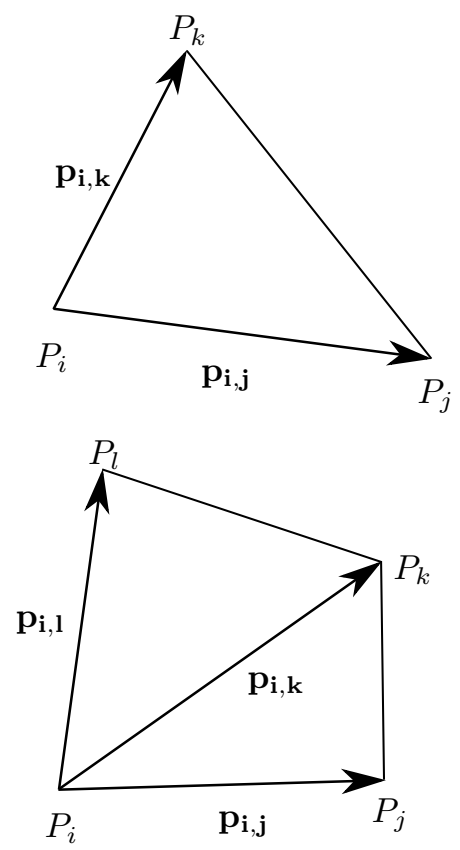

Figure 4: Top: The bilateration problem. Bottom: Concatenation of two bilaterations.

The bilateration problem consists in finding the feasible locations of a point, say $P_{k}$, given its distances to two other points, say $P_{i}$ and $P_{j}$, whose locations are known. Then, according to Fig. 4(top), the result to this problem, in matrix form, can be expressed as:

$$
\mathbf{p}_{i, k}=\mathbf{Z}_{i, j, k} \mathbf{p}_{i, j}
$$

where $\mathbf{p}_{i, j}=\overrightarrow{P_{i} P_{j}}$ and

$$
\mathbf{Z}_{i, j, k}=\frac{1}{2 s_{i, j}}\left[\begin{array}{cc}
s_{i, j}+s_{i, k}-s_{j, k} & -4 A_{i, j, k} \\
4 A_{i, j, k} & s_{i, j}+s_{i, k}-s_{j, k}
\end{array}\right]
$$

is called a bilateration matrix, with $s_{i, j}=\left\|\mathbf{p}_{i, j}\right\|^{2}$, the squared distance between $P_{i}$ and $P_{j}$, and

$$
A_{i, j, k}= \pm \frac{1}{4} \sqrt{\left(s_{i, j}+s_{i, k}+s_{j, k}\right)^{2}-2\left(s_{i, j}^{2}+s_{i, k}^{2}+s_{j, k}^{2}\right)} \text {, }
$$

the oriented area of $\triangle P_{i} P_{j} P_{k}$ which is defined as positive if $P_{k}$ is to the left of vector $\mathbf{p}_{i, j}$, and negative otherwise. The interested reader is addressed to [20] for a derivation of (1).

Given the triangle in Fig. 4 (top), it is possible to compute six different bilaterations. By algebraically manipulating the obtained results, it is possible to prove that:

$$
\begin{aligned}
& \mathbf{Z}_{i, j, k}=\mathbf{I}-\mathbf{Z}_{j, i, k} \\
& \mathbf{Z}_{i, j, k}=\left(\mathbf{Z}_{i, k, j}\right)^{-1} \\
& \mathbf{Z}_{i, j, k}=-\mathbf{Z}_{k, j, i} \mathbf{Z}_{j, i, k} .
\end{aligned}
$$

Moreover, it can be observed that the product of two bilateration matrices is commutative. Then, it is easy to prove that the set of bilateration matrices, i.e., matrices of the form $\left(\begin{array}{cc}a & -b \\ b & a\end{array}\right)$, constitute a commutative group under the product and addition operations.

Another important property, that will be useful later, comes from the fact that if $\mathbf{v}=\mathbf{Z w}$, where $\mathbf{Z}$ is a bilateration matrix, then $\|\mathbf{v}\|^{2}=\operatorname{det}(\mathbf{Z})\|\mathbf{w}\|^{2}$.

Now, let us consider the two triangles sharing one edge depicted in Fig. 4 (bottom). Then, $\mathbf{p}_{i, l}$ can be expressed in terms of $\mathbf{p}_{i, j}$ by applying two consecutive bilaterations, as

$$
\mathbf{p}_{i, l}=\mathbf{Z}_{i, k, l} \mathbf{p}_{i, k}=\mathbf{Z}_{i, k, l} \mathbf{Z}_{i, j, k} \mathbf{p}_{i, j}
$$

Actually, a vector involving any two different points in the set $\left\{P_{i}, P_{j}, P_{k}, P_{l}\right\}$ can be expressed in function of $\mathbf{p}_{i, j}$ using bilateration matrices. For example,

$$
\mathbf{p}_{j, l}=\mathbf{p}_{i, l}-\mathbf{p}_{i, j}=\left(\mathbf{Z}_{i, k, l} \mathbf{Z}_{i, j, k}-\mathbf{I}\right) \mathbf{p}_{i, j}
$$

Therefore, the squared distance between $P_{j}$ and $P_{l}$ can be obtained as:

$$
s_{j, l}=\operatorname{det}\left(\mathbf{Z}_{i, k, l} \mathbf{Z}_{i, j, k}-\mathbf{I}\right) s_{i, j}
$$

If this result is compared to the one presented for example in [21, pp. 65-69], one starts to appreciate the ability of bilateration matrices to represent the solution of complex problems in a very compact form. This result can be extended to a strip of triangles -i.e., a series of connected triangles that share one edge with one neighbor and another with the next - to obtain the squared distance between any couple of its vertices. As an example, let us suppose that we are interested in finding $\mathbf{p}_{1,3}$ as a function of $\mathbf{p}_{2,4}$ for the strip of three triangles $\left\{\triangle P_{1} P_{10} P_{2}, \triangle P_{2} P_{10} P_{4}, \triangle P_{10} P_{3} P_{4}\right\}$ appearing in Fig. 5 h. In this case,

$$
\begin{aligned}
\mathbf{p}_{1,3} & =-\mathbf{p}_{2,1}+\mathbf{p}_{2,4}+\mathbf{p}_{4,3} \\
& =-\mathbf{Z}_{2,10,1} \mathbf{p}_{2,10}+\mathbf{p}_{2,4}+\mathbf{Z}_{4,10,3} \mathbf{p}_{4,10} \\
& =-\mathbf{Z}_{2,10,1} \mathbf{Z}_{2,4,10} \mathbf{p}_{2,4}+\mathbf{p}_{2,4}+\mathbf{Z}_{4,10,3} \mathbf{p}_{4,10} \\
& =\left(-\mathbf{Z}_{2,10,1} \mathbf{Z}_{2,4,10}+\mathbf{I}-\mathbf{Z}_{4,10,3} \mathbf{Z}_{4,2,10}\right) \mathbf{p}_{2,4}
\end{aligned}
$$

Therefore, the squared distance $s_{1,3}$ can be expressed as:

$$
s_{1,3}=\operatorname{det}\left(\boldsymbol{\Omega}_{1}\right) s_{2,4},
$$

where $\boldsymbol{\Omega}_{1}=-\mathbf{Z}_{2,10,1} \mathbf{Z}_{2,4,10}+\mathbf{I}-\mathbf{Z}_{4,10,3} \mathbf{Z}_{4,2,10}$. 


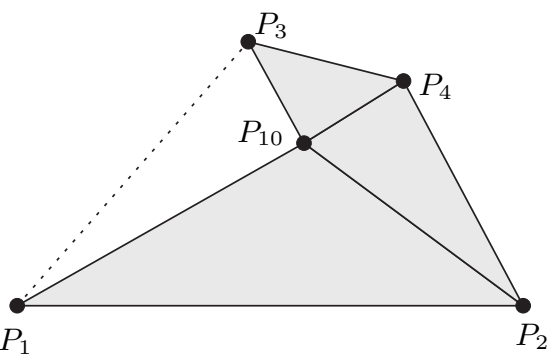

(a)

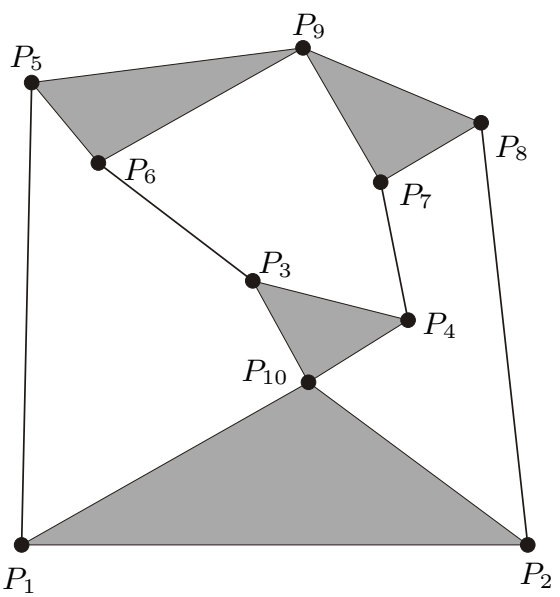

(d)

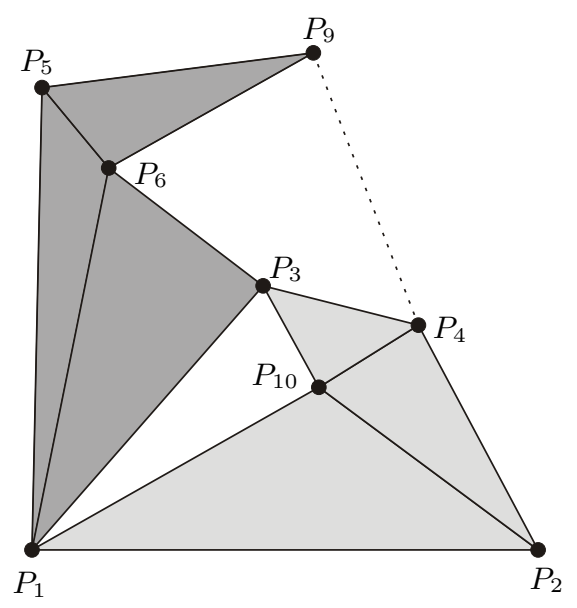

(b)

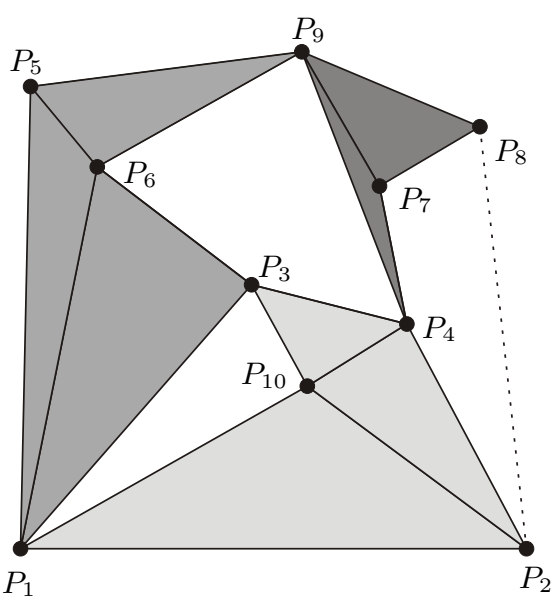

(c)

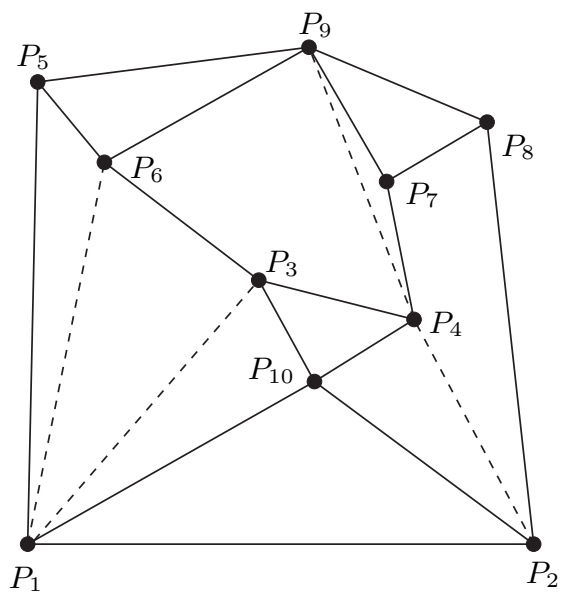

(e)

Figure 5: (a) In the strip of triangles $\left\{\triangle P_{1} P_{10} P_{2}, \triangle P_{2} P_{10} P_{4}, \triangle P_{10} P_{3} P_{4}\right\}, s_{1,3}$ can be obtained from bilaterations. (b) After affixing the strip of triangles $\left\{\triangle P_{1} P_{6} P_{3}, \triangle P_{1} P_{5} P_{6}, \triangle P_{6} P_{5} P_{9}\right\}$ to the previous one, $s_{4,9}$ can also be obtained using bilaterations. (c) Likewise, $s_{2,6}$ can be obtained after affixing the strip of triangles $\left\{\triangle P_{4} P_{9} P_{7}, \triangle P_{7} P_{9} P_{8}\right\}$. (d) A double butterfly linkage. (e) If the lengths of dotted segments were known, this double butterfly linkage would be equivalent to the obtained structure resulting from attaching three strips of triangles.

The possibility of computing squared distances that involve arbitrary couples of vertices, using sequences of bilaterations, is not limited to strips of triangles. This can also be applied, for example, to two strips sharing two arbitrary vertices. To exemplify this, let us suppose that we are interested in finding $\mathbf{p}_{4,9}$ as a function of $\mathbf{p}_{2,4}$ after attaching the strip of triangles defined by $\left\{\triangle P_{1} P_{6} P_{3}, \triangle P_{1} P_{5} P_{6}, \triangle P_{6} P_{5} P_{9}\right\}$ to the previous strip so that they share vertices $P_{1}$ and $P_{3}$ [see Fig. 5(b)]. Then,

$$
\begin{aligned}
\mathbf{p}_{4,9}= & -\mathbf{p}_{2,4}+\mathbf{p}_{2,1}+\mathbf{p}_{1,6}+\mathbf{p}_{6,9} \\
= & -\mathbf{p}_{2,4}+\mathbf{Z}_{2,10,1} \mathbf{p}_{2,10}+\mathbf{p}_{1,6}+\mathbf{Z}_{6,5,9} \mathbf{p}_{6,5} \\
= & \left(-\mathbf{I}+\mathbf{Z}_{2,10,1} \mathbf{Z}_{2,4,10}\right) \mathbf{p}_{2,4}+\left(\mathbf{I}-\mathbf{Z}_{6,5,9}\right. \\
& \left.\mathbf{Z}_{6,1,5}\right) \mathbf{p}_{1,6} \\
= & \left(-\mathbf{I}+\mathbf{Z}_{2,10,1} \mathbf{Z}_{2,4,10}\right) \mathbf{p}_{2,4}+\left(\mathbf{I}-\mathbf{Z}_{6,5,9} \mathbf{Z}_{6,1,5}\right) \\
& \mathbf{Z}_{1,3,6} \mathbf{p}_{1,3}
\end{aligned}
$$

$$
\begin{aligned}
= & \left(-\mathbf{I}+\mathbf{Z}_{2,10,1} \mathbf{Z}_{2,4,10}\right) \mathbf{p}_{2,4}+\left(\mathbf{I}-\mathbf{Z}_{6,5,9} \mathbf{Z}_{6,1,5}\right) \\
& \mathbf{Z}_{1,3,6} \boldsymbol{\Omega}_{1} \mathbf{p}_{2,4} \\
= & \left(-\mathbf{I}+\mathbf{Z}_{2,10,1} \mathbf{Z}_{2,4,10}+\left(\mathbf{I}-\mathbf{Z}_{6,5,9} \mathbf{Z}_{6,1,5}\right)\right. \\
& \left.\mathbf{Z}_{1,3,6} \boldsymbol{\Omega}_{1}\right) \mathbf{p}_{2,4} .
\end{aligned}
$$

Therefore,

$$
s_{4,9}=\operatorname{det}\left(\boldsymbol{\Omega}_{2}\right) s_{2,4}
$$

where

$$
\begin{aligned}
\boldsymbol{\Omega}_{2}= & -\mathbf{I}+\mathbf{Z}_{2,10,1} \mathbf{Z}_{2,4,10}+\left(\mathbf{I}-\mathbf{Z}_{6,5,9} \mathbf{Z}_{6,1,5}\right) \\
& \mathbf{Z}_{1,3,6} \boldsymbol{\Omega}_{1}
\end{aligned}
$$

The process of adding a strip of triangles sharing two arbitrary vertices with the obtained structure can be iterated further. For example, we can now add the strip of triangles defined by $\left\{\triangle P_{4} P_{9} P_{7}, \triangle P_{7} P_{9} P_{8}\right\}$, as shown in Fig. 5 (c). In this case, we might be interested in obtaining $s_{2,8}$ as a 
function of $s_{2,4}$. To this end, we could compute

$$
\begin{aligned}
\mathbf{p}_{2,8} & =\mathbf{p}_{2,4}+\mathbf{p}_{4,9}+\mathbf{p}_{9,8} \\
& =\mathbf{p}_{2,4}+\left(\mathbf{I}-\mathbf{Z}_{9,7,8} \mathbf{Z}_{9,4,7}\right) \mathbf{p}_{4,9} \\
& =\left(\mathbf{I}+\left(\mathbf{I}-\mathbf{Z}_{9,7,8} \mathbf{Z}_{9,4,7}\right) \boldsymbol{\Omega}_{2}\right) \mathbf{p}_{2,4} .
\end{aligned}
$$

Therefore,

$$
s_{2,8}=\operatorname{det}\left(\boldsymbol{\Omega}_{3}\right) s_{2,4}
$$

where

$$
\boldsymbol{\Omega}_{3}=\mathbf{I}+\left(\mathbf{I}-\mathbf{Z}_{9,7,8} \mathbf{Z}_{9,4,7}\right) \boldsymbol{\Omega}_{2} .
$$

Then, if $s_{2,8}$ is fixed, equation (11) can be seen as a closure condition: it is satisfied if, and only if, the resulting structure can be assembled with the specified distances. This idea can be applied to solve the position analysis problem of linkages, as shown next for the double butterfly linkage.

\section{Tracing the double butterfly linkage config- uration space}

The double butterfly linkage has one of the sixteen topologies available for 8-bar single-degree-of-freedom linkages [19]. In the context of classical kinematics of mechanisms, the input-output problem for this linkage leads to either sixteenth order or eighteenth order polynomials depending on the selected fix and input links [2, 4, 22]. This input-output problem, that was solved using continuation in [23], is equivalent to the position analysis problem of the seven-link Baranov trusses of type II and III [20, 24]. A polynomial equation for the path of a point located in a coupler link of the double butterfly linkage was presented in [18] for the first time. The resulting polynomial was shown to be, at most, of fortyeighth order. A sampled plot of this curve is presented in [25]. The interested reader is referred to [26] for more details on this mechanism.

Fig. 5(d) shows a double butterfly linkage. It consists of four binary links and four ternary links with three independent loops. The centers of the revolute joints of the binary links define the line segments $\overline{P_{1} P_{5}}, \overline{P_{3} P_{6}}, \overline{P_{4} P_{7}}$, and $\overline{P_{2} P_{8}}$, and those for the ternary links define the triangles $\triangle P_{1} P_{10} P_{2}, \triangle P_{10} P_{3} P_{4}, \triangle P_{6} P_{5} P_{9}$, and $\triangle P_{7} P_{9} P_{8}$. Instead of computing its configuration space in terms of joint angles through loop-closure equations, we will use squared distances in strips of triangles to compute the set of values of $s_{2,4}$ and $s_{1,6}$ compatible with all binary and ternary link side lengths.

It can be verified that, if the distances $s_{1,3}, s_{1,6}, s_{4,9}$, and $s_{2,4}$ of the double butterfly linkage in Fig. 5(d) were fixed, the structure in Fig. 5 c) would be obtained [Fig.5(e)]. Then, if we rewrite equations (9), (10), and (11), leaving these distances as variables, we get the following system of equations:

$$
\begin{aligned}
& s_{1,3}=f_{1}\left(s_{2,4}\right)=\operatorname{det}\left(\boldsymbol{\Omega}_{1}\right) s_{2,4} \\
& s_{4,9}=f_{2}\left(s_{2,4}, s_{1,6}, s_{1,3}\right)=\operatorname{det}\left(\boldsymbol{\Omega}_{2}\right) s_{2,4} \\
& s_{2,8}=f_{3}\left(s_{2,4}, s_{1,6}, s_{1,3}, s_{4,9}\right)=\operatorname{det}\left(\boldsymbol{\Omega}_{3}\right) s_{2,4} .
\end{aligned}
$$

Computing a resultant from the above triangular system becomes a trivial task that yields a scalar radical equation in two variables: $s_{2,4}$ and $s_{1,6}$.

By expanding (12c), we get

$$
s_{2,8}=\frac{1}{s_{1,6} s_{1,3} s_{4,9}} \Psi
$$

where

$$
\begin{aligned}
\Psi & =\Psi_{1}+\Psi_{2} A_{2,4,10}+\Psi_{3} A_{1,3,6}+\Psi_{4} A_{1,6,5}+\Psi_{5} A_{4,9,7} \\
& +\Psi_{6} A_{2,4,10} A_{1,3,6}+\Psi_{7} A_{2,4,10} A_{1,6,5}+\Psi_{8} A_{2,4,10} A_{4,9,7} \\
& +\Psi_{9} A_{1,3,6} A_{1,6,5}+\Psi_{10} A_{1,3,6} A_{4,9,7}+\Psi_{11} A_{1,6,5} A_{4,9,7} \\
& +\Psi_{12} A_{2,4,10} A_{1,3,6} A_{1,6,5}+\Psi_{13} A_{2,4,10} A_{1,3,6} A_{4,9,7} \\
& +\Psi_{14} A_{2,4,10} A_{1,6,5} A_{4,9,7}+\Psi_{15} A_{1,3,6} A_{1,6,5} A_{4,9,7} \\
& +\Psi_{16} A_{2,4,10} A_{1,3,6} A_{1,6,5} A_{4,9,7}
\end{aligned}
$$

with $\Psi_{i}, i=1, \ldots, 16$, being polynomials in $s_{2,4}, s_{1,6}, s_{1,3}$, and $s_{4,9}$, and $A_{2,4,10}, A_{1,3,6}, A_{1,6,5}$, and $A_{4,9,7}$, the oriented areas of $\triangle P_{2} P_{4} P_{10}, \triangle P_{1} P_{6} P_{3}, \triangle P_{1} P_{6} P_{5}$, and $\triangle P_{4} P_{9} P_{7}$, respectively, and substituting (12a) and (12b) which account for the unknown squared distances $s_{1,3}$ and $s_{4,9}$.

Equation (13) is the closure condition for the double butterfly linkage. This equation contains four variable oriented areas, namely, $A_{2,4,10}, A_{1,3,6}, A_{1,6,5}$, and $A_{4,9,7}$. Each of these oriented areas involves a radical. By iteratively isolating one radical on one side of the equation and then squaring both sides of the equation, it is possible to transform equation (13) to polynomial form. Nevertheless, it is advantageous to keep it in its current form, not only because of its compactness compared to the polynomial form, but because, and most importantly, it provides a decomposition of the configuration space of the double butterfly linkage into sixteen varieties, one for each combination of signs of the oriented areas $A_{2,4,10}, A_{1,3,6}, A_{1,6,5}$ and $A_{4,9,7}$. Therefore, the configuration space of the double butterfly linkage can be decomposed into sixteen components in the space defined by $\left\{s_{1,6}, s_{2,4}\right\}$.

The advantage of this decomposition is that the resulting components can be easily traced. In the example presented in the following section, it is shown how all the crunodes appear as intersections of two different components, and how all the cusps appear when mapping these traced components onto the workspace. Starting from a given configuration in a component, the tracing process would proceed till the starting point is again reached or when it arrives at a point where one of the defining oriented areas vanishes. Then, the tracing process would have to skip to another component: the one defined by the same oriented area signs except for the area that vanishes whose sign has to be changed.

\section{Example}

According to the notation used in Fig. 5(d), let us set $s_{1,2}=169, s_{1,5}=145, s_{1,10}=65, s_{2,8}=200, s_{2,10}=52$, $s_{3,4}=5, s_{3,6}=50, s_{3,10}=5, s_{4,7}=36, s_{4,10}=10$, $s_{5,6}=5, s_{5,9}=53, s_{6,9}=34, s_{7,8}=10, s_{7,9}=5$, and $s_{8,9}=25$. Using triangular inequalities, $s_{2,4}$ can be bound to lie in the interval $[62-4 \sqrt{13} \sqrt{10}, 62+4 \sqrt{13} \sqrt{10}]$. Fig. 6(top) shows the root locus of (13). This root locus 

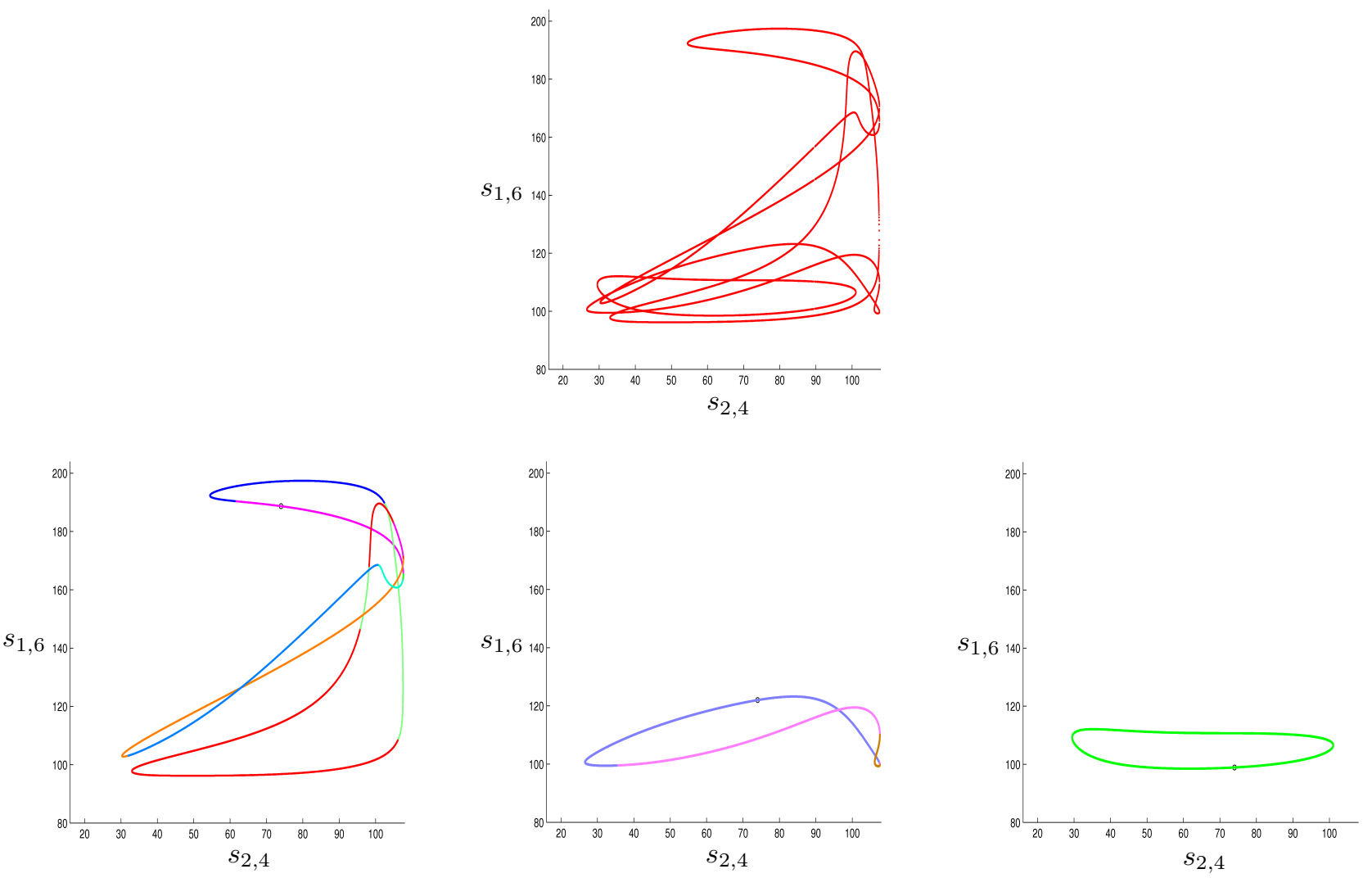

Figure 6: Top: The real solution set of equation (13) in the plane defined by $s_{2,4}$ and $s_{1,6}$ for sampled values of $s_{2,4}$. Bottom: From left to right, the connected components of the configuration space traced when starting from the initial configurations $s_{2,4}=74, s_{1,6}=188.68$, and $A_{2,4,10}>0, A_{1,3,6}<0, A_{1,6,5}<0$, and $A_{4,9,7}<0, s_{2,4}=74, s_{1,6}=122$, and $A_{2,4,10}>0$, $A_{1,3,6}>0, A_{1,6,5}>0$, and $A_{4,9,7}<0$, and $s_{2,4}=74, s_{1,6}=98.92$, and $A_{2,4,10}<0, A_{1,3,6}<0, A_{1,6,5}<0$, and $A_{4,9,7}<0$, respectively.

has been obtained by clearing the radicals in (13) to obtain a polynomial equation in $s_{2,4}$ and $s_{1,6}$ and then solving this equation for sampled values of $s_{2,4}$ at increments of $\frac{1}{100}$. The result contains no information on the connectivity of each sample to its neighbors. Actually, finding this connectivity is the difficult point but any of the obtained samples can be used as a starting point for a tracing algorithm. The sampled curve has been included here for comparison purposes with the results obtained by tracing as shown next.

Let us suppose that we are interested in tracing the configuration space followed by the linkage from the following three initial configurations:

1. $s_{2,4}=74, s_{1,6}=188.68$, and $A_{2,4,10}>0, A_{1,3,6}<0$, $A_{1,6,5}<0$, and $A_{4,9,7}<0$,

2. $s_{2,4}=74, s_{1,6}=122$, and $A_{2,4,10}>0, A_{1,3,6}>0$, $A_{1,6,5}>0$, and $A_{4,9,7}<0$,

3. $s_{2,4}=74, s_{1,6}=98.92$, and $A_{2,4,10}<0, A_{1,3,6}<0$, $A_{1,6,5}<0$, and $A_{4,9,7}<0$.

The results using the procedure discussed in the previous section appear in Figure 6(bottom), from left to right, respectively. In the three plots, colors indicate the signs of the oriented areas according to Table 1

\begin{tabular}{ccccc}
\hline Color & $A_{2,4,10}$ & $A_{1,3,6}$ & $A_{1,6,5}$ & $A_{4,9,7}$ \\
\hline & - & - & - & - \\
- & - & - & + \\
& - & + & - & - \\
- & + & - & + \\
- & + & + & + \\
+ & - & - & - \\
+ & - & - & + \\
+ & + & - & - \\
+ & + & - & + \\
+ & + & + & - \\
+ & + & + & + \\
\hline
\end{tabular}

Table 1: Code of colors used in Figs. 6 and 7 for the signs of $A_{2,4,10}, A_{1,3,6}, A_{1,6,5}$, and $A_{4,9,7}$.

In order to determine the coupler curve of a selected tracer of the linkage using the computed configuration space, we proceed to calculate the position of the linkage's revolute pair centers using bilateration. Let us suppose that $\triangle P_{1} P_{2} P_{10}$ is the fixed link. Then, for example, we can set $\mathbf{p}_{1}=(4,0)^{T}, \mathbf{p}_{2}=(17,0)^{T}$, and $\mathbf{p}_{10}=(11,4)^{T}$, and the path traced by $P_{3}, P_{4}, P_{5}, P_{6}, P_{7}, P_{8}$, and $P_{9}$ can be obtained by replacing each previously computed configuration 
space point in the sequence of bilaterations given by:

$$
\begin{aligned}
\mathbf{p}_{2,4} & =\mathbf{Z}_{2,10,4} \mathbf{p}_{2,10} \\
\mathbf{p}_{10,3} & =\mathbf{Z}_{10,4,3} \mathbf{p}_{10,4}, \\
\mathbf{p}_{1,6} & =\mathbf{Z}_{1,3,6} \mathbf{p}_{1,3}, \\
\mathbf{p}_{1,5} & =\mathbf{Z}_{1,6,5} \mathbf{p}_{1,6} \\
\mathbf{p}_{5,9} & =\mathbf{Z}_{5,6,9} \mathbf{p}_{5,6} \\
\mathbf{p}_{4,7} & =\mathbf{Z}_{4,9,7} \mathbf{p}_{4,9} \\
\mathbf{p}_{7,8} & =\mathbf{Z}_{7,9,8} \mathbf{p}_{7,9}
\end{aligned}
$$

Fig. 7(top) shows the path followed by $P_{9}$ from the first initial configuration. It can be observed how the mapping from configuration space to workspace is surjective (two points of the configuration space can be mapped onto the same point in the workspace) and how this fact is actually the underlying reason that makes coupler curves so difficult to be traced directly in the linkage workspace. The zoomedin areas in Fig. 7 top) present this effect by showing how two overlapping branches next to a ramphoid cusp, which leads to a reciprocating motion of the linkage, and a nearquadruple point are generated. Similar situations arise for the path followed by $P_{9}$ from the second initial configuration. In this case a cusp and a tacnode can be identified [Fig. 7(center)]. The curve traced when starting from the third initial configuration appears in [Fig. 7(bottom)]. Observe how in this case a smooth simple curve in the configuration space maps onto the linkage workspace as a curve with several singular points in a reduced area which would be very difficult to be directly traced using a standard predictorcorrector procedure without highly increasing its resolution.

If we were interested in the curve traced by a coupler point different from the revolute pair centers, we could compute its location by introducing one extra bilateration with reference to the revolute pair centers of the corresponding coupler link.

For the curves traced in a different kinematic inversion of the linkage, we simply have to calculate the Euclidean transformation between the constant values of the corresponding fixed link and the values computed with the above set of bilaterations, and use it to recompute the values for the other revolute pair centers. With this simple procedure, the coupler curves of any kinematic inversion of the double butterfly linkage can be computed.

As it has been shown in this example, the main advantage of the proposed method for tracing the coupler curves is that the configuration space of single-degree-of-freedom linkages can be decomposed into easy-to-trace branches. Actually, in the presented example all singularities arise when mapping these branches onto the linkage workspace.

\section{Other single-degree-of-freedom linkages}

The proposed method for tracing coupler curves can be easily applied to any single-degree-of-freedom planar linkage. It can be verified that tracing the coupler curves of the 4-bar linkage and the two 6-bar linkages - the Watt and the Stephenson linkages - becomes trivial because their configuration spaces correspond to ranges of a single distance, one range for each combination of sign of two or three oriented areas, depending on the case. A similar situation occurs for twelve of the sixteen possible topologies for singledegree-of-freedom 8-bar linkages. In these cases, four oriented areas are needed. For the remaining four topologies -in which the double butterfly linkage is included- the one-dimensional configuration space is embedded in a twodimensional distance space. Since the sign of four oriented areas are needed in all these four cases to uniquely identify a configuration, the configuration space is naturally decomposed into 16 components. Table 2 presents the equations representing the corresponding configuration spaces for these four cases.

\section{Conclusion}

The current approaches for tracing the coupler curves of plane mechanisms provide a rapid algebraization of the problem thus becoming blind to the underlying geometry. A new Distance Geometry approach that first computes the linkage configuration space embedded in a space of squared distances and then maps it onto the linkage workspace has been presented. The used formulation involves products, additions, and square roots. The presence of square roots permits a more compact representation than the standard techniques based on polynomials. Square roots actually play a fundamental role in the presented approach because their sign represent the orientation of triangles formed by sets of three joints of the linkage thus retaining important geometric information. Configuration spaces are thus decomposed into components for which the signs of the oriented areas of the involved triangles remain invariant. This decomposition, besides providing a new insight in the analysis of coupler curves, avoids most of the possible drifts that could arise when using a standard predictor-corrector method directly in the linkage workspace. In all the experiments we have carried out with the proposed method, all individual components, defined by constant sign areas, of the configuration space are free from singularities. As a result, tracing them is an easy task. We actually conjecture that, in general, these components cannot self-intersect. Unfortunately, a formal proof or a counterexample remains elusive despite our best efforts. This is a point that deserves further attention.

\section{Acknowledgments}

We gratefully acknowledge the financial support of the Spanish Ministry of Economy and Competitiveness, under the I+D project DPI2011-13208-E, and the Colombian Ministry of Communications and Colfuturo through the ICT National Plan of Colombia.

\section{References}

[1] Freudenstein, F., 1962. "On the variety of motions generated by mechanisms". Journal of Engineering for Industry, 39(February), pp. $156-160$. 


\begin{tabular}{ccc}
\hline Linkage & $\begin{array}{c}\text { Distance space } \\
\text { and oriented areas }\end{array}$ & Closure conditions \\
\hline
\end{tabular}

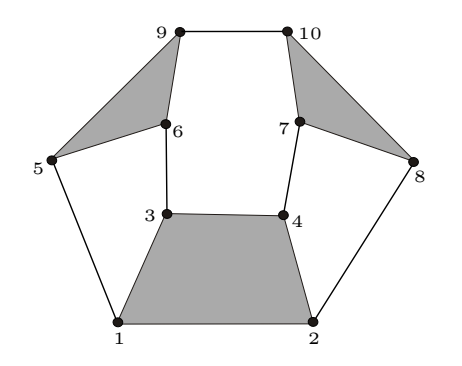

$$
\begin{aligned}
& \left(s_{1,6}, s_{2,7}\right) \quad s_{9,10}=f\left(s_{1,6}, s_{2,7}\right) \\
& A_{1,6,3}, A_{1,6,5}, \quad=\operatorname{det}\left(-\mathbf{I}+\mathbf{Z}_{6,5,9} \mathbf{Z}_{6,1,5}+\mathbf{Z}_{1,3,4} \mathbf{Z}_{1,6,3}-\mathbf{Z}_{4,1,2} \mathbf{Z}_{1,3,4} \mathbf{Z}_{1,6,3}\right. \\
& \left.A_{2,4,7}, A_{2,7,8} \quad-\left(\mathbf{I}-\mathbf{Z}_{7,8,10} \mathbf{Z}_{7,2,8}\right) \mathbf{Z}_{4,2,7} \mathbf{Z}_{4,1,2} \mathbf{Z}_{1,3,4} \mathbf{Z}_{1,6,3}\right) s_{1,6}
\end{aligned}
$$

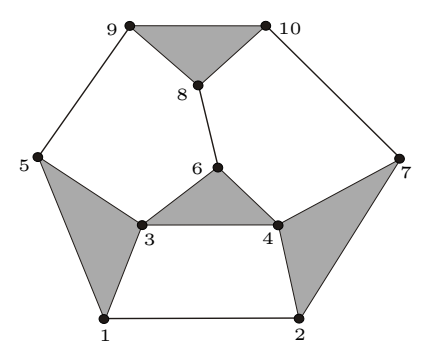

$$
\begin{aligned}
\left(s_{1,4}, s_{6,9}\right) \quad= & \operatorname{det}\left(\mathbf{Z}_{4,2,7} \mathbf{Z}_{4,1,2}-\mathbf{Z}_{4,3,6} \mathbf{Z}_{4,1,3}-\left(\mathbf{I}-\mathbf{Z}_{9,8,10} \mathbf{Z}_{9,6,8}\right)\right. \\
& \left.\mathbf{Z}_{6,5,9} \boldsymbol{\Omega}_{1}\right) s_{1,4}
\end{aligned}
$$

$A_{1,4,3}, A_{1,4,2}$

$A_{5,6,9}, A_{6,9,8} \quad s_{5,6}=f\left(s_{1,4}\right)=\operatorname{det}\left(\boldsymbol{\Omega}_{1}\right) s_{1,4}$

$$
=\operatorname{det}\left(-\mathbf{Z}_{1,3,5} \mathbf{Z}_{1,4,3}+\mathbf{I}-\mathbf{Z}_{4,3,6} \mathbf{Z}_{4,1,3}\right) s_{1,4}
$$

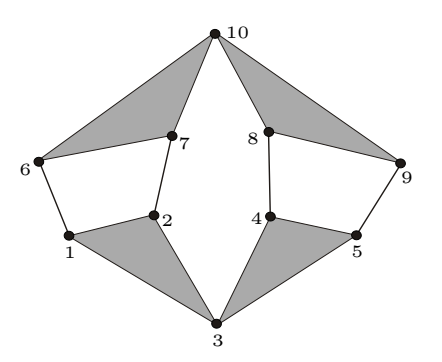

$$
\begin{aligned}
& \left(s_{1,7}, s_{4,10}\right) \quad s_{5,9}=f\left(s_{1,7}, s_{4,10}, s_{3,10}\right) \\
& A_{1,7,6}, A_{1,7,2}, \quad s_{3,10}=f\left(s_{1,7}\right)=\operatorname{det}\left(\boldsymbol{\Omega}_{1}\right) s_{1,7} \\
& A_{3,10,4}, A_{4,10,8}=\operatorname{det}\left(-\mathbf{Z}_{1,2,3} \mathbf{Z}_{1,7,2}+\mathbf{I}-\mathbf{Z}_{7,6,10} \mathbf{Z}_{7,1,6}\right) s_{1,7}
\end{aligned}
$$

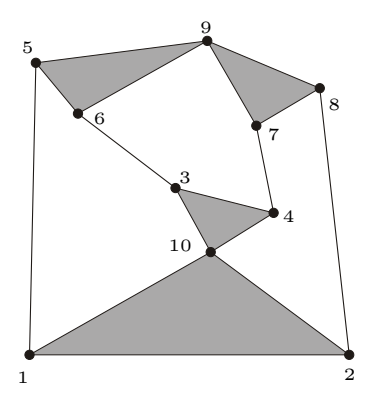

$$
\begin{aligned}
& s_{2,8}=f\left(s_{2,4}, s_{1,6}, s_{1,3}, s_{4,9}\right) \\
& =\operatorname{det}\left(\mathbf{I}+\left(\mathbf{I}-\mathbf{Z}_{9,7,8} \mathbf{Z}_{9,4,7}\right) \boldsymbol{\Omega}_{2}\right) s_{2,4} \\
& \left(s_{2,4}, s_{1,6}\right) \quad s_{4,9}=f\left(s_{2,4}, s_{1,6}\right)=\operatorname{det}\left(\boldsymbol{\Omega}_{2}\right) s_{2,4} \\
& A_{2,4,10}, A_{1,3,6} \text {, } \\
& =\operatorname{det}\left(-\mathbf{I}+\mathbf{Z}_{2,10,1} \mathbf{Z}_{2,4,10}+\left(\mathbf{I}-\mathbf{Z}_{6,5,9} \mathbf{Z}_{6,1,5}\right)\right. \\
& A_{1,6,5}, A_{4,9,7} \\
& \left.\mathbf{Z}_{1,3,6} \boldsymbol{\Omega}_{1}\right) s_{2,4} \\
& s_{1,3}=f\left(s_{2,4}\right)=\operatorname{det}\left(\boldsymbol{\Omega}_{1}\right) s_{2,4} \\
& =\operatorname{det}\left(-\mathbf{Z}_{2,10,1} \mathbf{Z}_{2,4,10}+\mathbf{I}-\mathbf{Z}_{4,10,3} \mathbf{Z}_{4,2,10}\right) s_{2,4}
\end{aligned}
$$

Table 2: The four 8-bar linkages whose configuration space can be embedded in a two-dimensional distance space. Since four oriented areas are needed in the four cases to uniquely identify a configuration, the corresponding configuration spaces are decomposed into 16 components. The equations representing the corresponding configuration spaces in implicit form are given on the right column. 

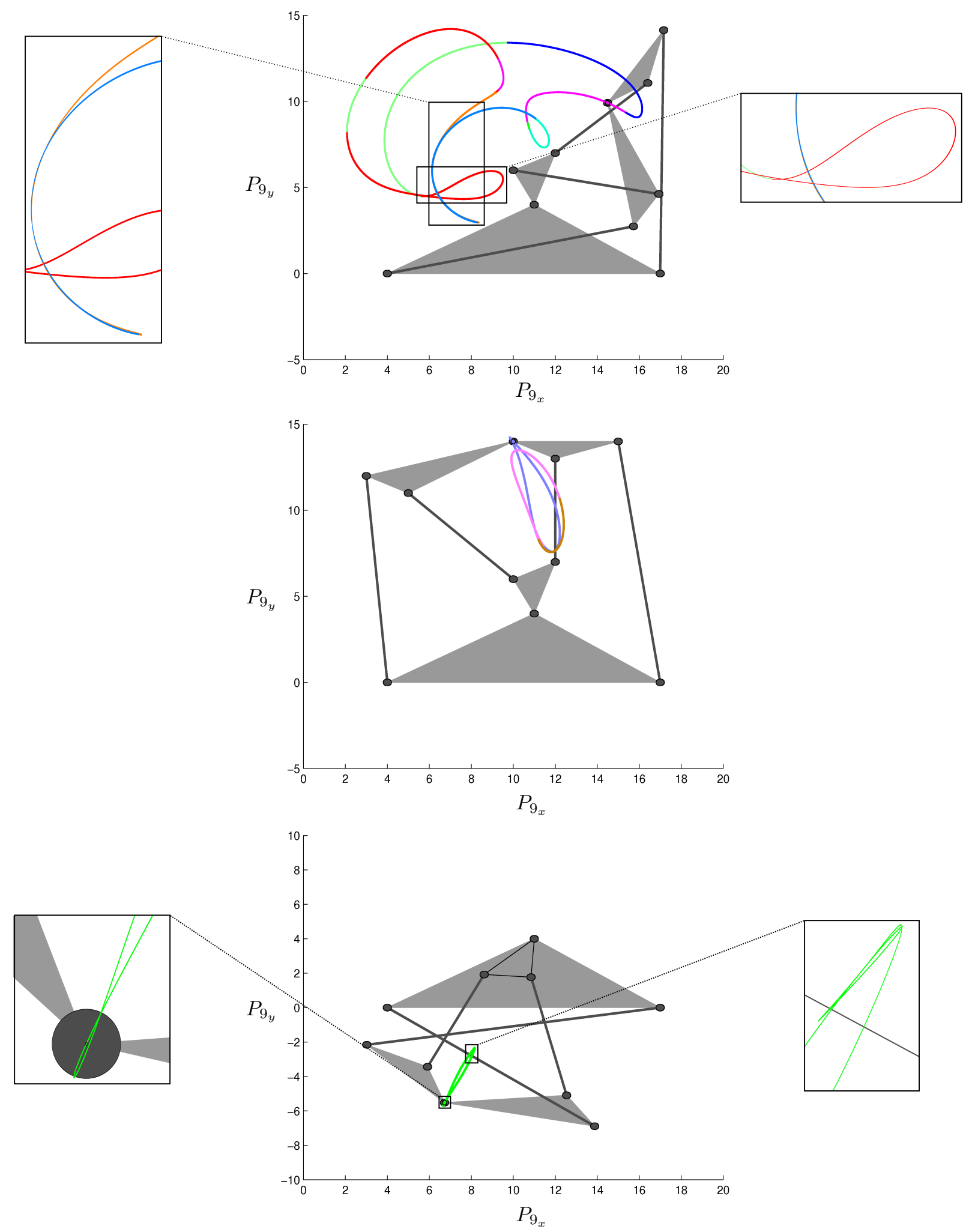

Figure 7: The paths followed by the revolute pair center $P_{9}$ from different initial configurations. Top: For the curve traced from the initial configuration $s_{2,4}=74, s_{1,6}=188.68$, and $A_{2,4,10}>0, A_{1,3,6}<0, A_{1,6,5}<0$, and $A_{4,9,7}<0$, zoomed-in areas show how, after mapping the configuration space onto the workspace, a ramphoid cusp and near-quadruple point are generated. Center: For the curve traced from the initial configuration $s_{2,4}=74, s_{1,6}=122$, and $A_{2,4,10}>0$, $A_{1,3,6}>0, A_{1,6,5}>0$, and $A_{4,9,7}<0$, a cusp and a tacnode can be identified. Bottom: For the curve traced from the initial configuration $s_{2,4}=74, s_{1,6}=98.92$, and $A_{2,4,10}<0, A_{1,3,6}<0, A_{1,6,5}<0$, and $A_{4,9,7}<0$, zoomed-in areas show how, after mapping the configuration space onto the workspace, several singular points are generated in a reduced region. 
[2] Nielsen, J., and Roth, B., 1999. "Solving the input/output problem for planar mechanisms". Journal of Mechanical Design, 121(2), pp. 206-211.

[3] Wampler, C., 1999. "Solving the kinematics of planar mechanisms". Journal of Mechanical Design, 121(3), pp. 387-391.

[4] Dhingra, A., Almadi, A., and Kohli, D., 2000. "Closedform displacement analysis of 8, 9 and 10-link mechanisms: Part I: 8-link 1-DOF mechanisms". Mechanism and Machine Theory, 35(6), pp. $821-850$.

[5] Lan, Z., Huijun, Z., and Liuming, L., 2002. "Kinematic decomposition of coupler plane and the study on the formation and distribution of coupler curves". Mechanism and Machine Theory, 37(1), pp. 115 - 126.

[6] Shirazi, K., 2006. "Symmetrical coupler curve and singular point classification in planar and spherical swinging-block linkages". Journal of Mechanical Design, 128(2), pp. 436-443.

[7] Allgower, E., and Georg, K., 2003. Introduction to Numerical Continuation Methods. SIAM.

[8] Sommese, A., and Wampler, C., 2005. The Numerical Solution of Systems of Polynomials Arising in Engineering and Science. World Scientific.

[9] Hernandez, A., and Petuya, V., 2004. "Position analysis of planar mechanisms with R-pairs using a geometrical-iterative method". Mechanism and Machine Theory, 39(2), pp. 133 - 152.

[10] Gomes, A., Voiculescu, I., Jorge, J., Wyvill, B., and Galbraith, C., 2009. Implicit Curves and Surfaces: Mathematics, Data Structures and Algorithms, 1st ed. Springer Publishing Company, Incorporated.

[11] Lambert, J., 1991. Numerical Methods for Ordinary Differential Systems: The Initial Value Problem. John Wiley and Sons.

[12] Ting, K., Xue, C., Wang, J., and Currie, K., 2009. "Stretch rotation and complete mobility identification of Watt six-bar chains". Mechanism and Machine Theory, 44(10), pp. $1877-1886$.

[13] Watanabe, K., and Katoh, H., 2004. "Identification of motion domains of planar six-link mechanisms of the Stephenson-type". Mechanism and Machine Theory, 39(10), pp. 1081 - 1099.

[14] Ting, K., and Dou, X., 1996. "Classification and branch identification of Stephenson six-bar chains". Mechanism and Machine Theory, 31(3), pp. 283 - 295.

[15] Wang, J., Ting, K., and Xue, C., 2010. "Discriminant method for the mobility identification of single degree-of-freedom double-loop linkages". Mechanism and Machine Theory, 45(5), pp. 740 - 755.

[16] Morgado, J., and Gomes, A., 2004. "A derivative-free tracking algorithm for implicit curves with singularities”. In International Conference on Computational Science, pp. 221-228.

[17] Hunt, K., 1978. Kinematic Geometry of Mechanisms. Clarendon Press, Oxford.

[18] Pennock, G., and Hasan, A., 2002. "A polynomial equation for a coupler curve of the double butterfly linkage". Journal of Mechanical Design, 124(1), pp. 39-46.
[19] McCarthy, J., and Soh, G., 2011. Geometric Design of Linkages. Springer.

[20] Rojas, N., and Thomas, F., 2011. "Distance-based position analysis of the three seven-link Assur kinematic chains". Mechanism and Machine Theory, 46(2), pp. $112-126$.

[21] Graver, J., 2001. Counting on Frameworks: Mathematics to Aid the Design of Rigid Structures. The Mathematical Association of America.

[22] Wampler, C., 2001. "Solving the kinematics of planar mechanisms by Dixon determinant and a complexplane formulation". Journal of Mechanical Design, 123(3), pp. 382-387.

[23] Waldron, K., and Sreenivasan, S., 1996. "A study of the solvability of the position problem for multi-circuit mechanisms by way of example of the double butterfly linkage". Journal of Mechanical Design, 118(3), pp. 390-395.

[24] Rojas, N., and Thomas, F., 2012. "On closed-form solutions to the position analysis of Baranov trusses". Mechanism and Machine Theory, 50, pp. 179 - 196.

[25] Porta, J., Ros, L., Creemers, T., and Thomas, F., 2007. "Box approximations of planar linkage configuration spaces". Journal of Mechanical Design, 129(4), pp. 397-405.

[26] Pennock, G., and Raje, N., 2004. "Curvature theory for the double flier eight-bar linkage". Mechanism and Machine Theory, 39(7), pp. 665-679. 